\title{
Automatic detection of interfering seismic wavelets using fractal methods
}

\author{
Shabanali Gholamy ${ }^{1}$, Abdolrahim Javaherian ${ }^{1,3}$ and Abdolreza Ghods ${ }^{2}$ \\ ${ }^{1}$ Institute of Geophysics, University of Tehran, I.R. Iran \\ ${ }^{2}$ Institute for Advanced Studies in Basic Sciences, Zanjan, I.R. Iran \\ E-mail: shaban_ali_gholamy@yahoo.com,javaheri@ut.ac.ir and aghods@iasbs.ac.ir
}

Received 11 August 2007

Accepted for publication 2 July 2008

Published 8 August 2008

Online at stacks.iop.org/JGE/5/338

\begin{abstract}
Interference of reflections from upper and lower interfaces of geological units is a common source of interpretational ambiguity. This paper utilizes the fractal dimension of instantaneous seismic attributes to study this interfering phenomenon. Using a 1D synthetic seismic trace, we examine the divider, Hurst, spectral and variance fractal methods. Since each method utilizes a moving window technique as part of its analysis, a series of optimum window lengths needs to be determined. The four methods are applied to the instantaneous amplitude, phase and frequency attributes of a series of 1D synthetic seismic traces. To study wavelet interference on 2D seismic sections, we generate four 2D synthetic seismic sections by convolving a zero phase and a minimum phase wavelet with two different wedge models. For the first model, the reflection coefficient of the upper wedge interface is positive and the lower interface negative, whereas for the second model, the polarities are positive. Our results suggest that fractal analysis of the instantaneous phase, using the Hurst method, can separate two opposite polarity wavelets within a distance of $\lambda / 16$ or greater and of $\lambda / 4$ or greater when they have the same polarity. Our results are not sensitive to random noise when the signal-to-noise-ratio is greater than 10 .
\end{abstract}

Keywords: fractal dimension, Hurst method, instantaneous attributes, wavelet interference, thin layers

\section{Introduction}

Interfering wavelets are an important consideration in seismic interpretation. Surface seismic recordings contain information about the thickness and the seismic impedance of subsurface geological units. Meissner and Meixner (1969) demonstrated that seismic waves reflected from the upper and lower interfaces of a thin geological layer interfere and construct a new pattern. Taner et al (1979) used analytic seismic traces for calculating instantaneous seismic attributes. Barnes (1993) introduced the idea of using instantaneous attributes to study interference of seismic wavelets.

Manual picking of seismic reflectors is a laborious task. Fractal methods facilitate the automatic picking of reflectors by simulating parts of the complex human intelligence used for their manual detection. Manual picking involves an operator's

\footnotetext{
3 Author to whom correspondence should be addressed.
}

decision for detecting sudden changes in the amplitude, frequency and phase in seismic traces. The fractal dimension of a signal is related to its complexity. The complex parts of a signal have a larger fractal dimension. The fractal dimension is measured over a moving time window of pre-determined size.

The application of fractal analysis in the auto-picking process of seismic horizons is a new concept. Using the divider method, Boschetti et al (1996) demonstrated picking of the first arrivals on seismic traces. Similarly, Jiao and Moon (2000) demonstrated picking on the onset of refracted signals in real and synthetic earthquake traces, using the variance fractal dimension (VFD). Nath and Dewangan (2002) used the divider and Hurst methods on instantaneous seismic attributes to detect the upper and lower interfaces of rock formations on seismic traces. No study has yet been made on the ability of the estimation of fractal dimension method to resolve layers 
with different thicknesses, and neither has the success rate of methods other than the divider and Hurst methods been calculated. Gholamy et al (2006) used the divider, Hurst, spectral and VFD fractal dimension methods to detect wavelet interference in seismic sections.

In this paper, we examine if the divider, Hurst, spectral and VFD fractal dimension methods can resolve thin layers in a seismic section which contain high levels of wavelet interference and random noise. A seismic trace is a complex object which cannot be uniquely described by only one parameter as its fractal dimension. Depending on the method used, the estimated fractal dimension can be related to the roughness, correlation length, power spectrum and variance of a signal. Since fractal dimensions measured by different methods are related to different properties of a signal, their values for a given signal may be different. Also, the computational cost and the error levels associated with a particular fractal method vary depending on the method used in fractal analysis. We use the four fractal dimension methods mentioned above on the instantaneous amplitude, instantaneous phase, and instantaneous frequency of seismic traces. The fractal dimension is estimated in a moving time window, while for each method, the optimum window size is determined. Then, we propose the optimum fractal method and the instantaneous attribute to resolve interfering reflectors. Synthetic seismic sections of two wedge models were used, in order to illustrate the ability of the proposed methodology in 2D seismic sections. Finally, the effects of random noise are examined.

\section{Methods of fractal dimension calculation}

\subsection{Divider method}

The 'structured walk technique' or 'divider method' (Hayward et al 1989, Kaye 1989) is used to measure the fractal dimension of self-similar curves such as the boundaries of continuous natural shapes (Mandelbrot 1967, Turcotte 1997). The divider method measures the length of a curve by approximating it with a number of straight lines, called 'steps' (Boschetti et al 1996). It is inherently impossible to measure a unique fractal dimension for a time series using the divider method. This is because time series are self-affine objects (Mandelbrot 1985, Turcotte 1997). The ratio of scales used for plotting the dependent variable against the independent (time) variable determines the size of the fractal dimension of the time series calculated by the divider method. Automatic detection of wavelets requires only the temporal variations of the fractal dimension and not its absolute values.

To monitor the temporal variations of a fractal dimension, the time series is scanned using a fixed-size moving time window and the fractal dimension is calculated for each window. First, we oversample the initial discrete time series five times, using a third-order spline. Next, the length of the time series within the chosen window is measured by dividers of different apertures. As the aperture decreases, the measured length increases as more details of the time series are detected. Measured lengths of the time series are plotted on a logarithmic scale against the divider apertures and a line is fitted to the points using the least squares method. The fractal dimension $D$ of the time series is related to the slope, $\alpha$, of the line (Mandelbrot 1967, Kennedy and Lin 1986):

$$
D=1-\alpha .
$$

The calculated fractal dimension is assigned to the last point of the moving time window because the starting point for the dividers is always the last point of a moving time window. Seismic traces are not fractal objects on all scales (Boschetti et al 1996); therefore, care must be taken in selecting the lower and upper limits of the divider aperture. Usually the logarithmic plot of the divider apertures versus the length shows a flat region in both the lower and upper ends of the divider apertures. Fitting a line through such a curve would give an incorrect fractal dimension for the part of the seismic signal that is selfaffine. To avoid this problem, we have selected a lower divider aperture length equal to the sampling interval of the seismic trace and an upper divider aperture length equal to one third of the moving window size. The distance between the divider lengths are selected so that the spread of points on the logarithmic axis of the divider aperture length becomes uniform. This will avoid the bias of the slope at smaller divider aperture lengths.

\subsection{Hurst method}

The Hurst method (Hurst et al 1965) is a method specifically designed to measure the fractal dimension of time series. The fractal dimension measured by this method is related to statistical correlations of independent variables (e.g., amplitude) with time. In this method, the fractal dimension is calculated by determining the range, $R$, of the data within windows of different sizes. The maximum difference observed in a window of a given size $(R)$ is normalized by dividing by the standard deviation of the data, $S$, within that window. For window sizes less than the length of a time series, the time series is segmented into an appropriate number of windows and then the averaged $R$ and $S$ values are calculated for that given window size. Time series are fractals provided that the logarithmic plot of $R / S$ versus the window size closely follows a straight sloping line. To have an unbiased fit, we draw the $\log$ of $R / S$ versus the equally binned logarithm of the window size.

The slope of the best fit line is calculated using the least squares method, while binning the logarithmic axis of the window size into equal bins. The slope of this line is known as the Hurst exponent (Hu). A Hurst exponent of $0.5<\mathrm{Hu}<1.0$ implies a persistence or correlation in the time series, whereas values of $0<\mathrm{Hu}<0.5 \mathrm{imply}$ an anti-persistence. A zero value for the Hurst exponent implies randomness. Hu can be related to the fractal dimension, $D$, by (Turcotte 1997)

$$
D=3-\mathrm{Hu} .
$$

To monitor the temporal variations of the Hurst exponent, we calculate $\mathrm{Hu}$ within a moving window. To make the Hurst 
exponent sensitive to the scaling of the signal at the end of a moving time window, we do not calculate an average $R$ and $S$ for all of the time window segments, but instead calculate the $R$ and $S$ values for the last time window segment within each moving time window.

\subsection{Power spectral method}

The fractal dimension calculated by this method is sensitive to the frequency content of the time series and is applied in the frequency domain. To monitor variations in the frequency content proportional to time, or simply the fractal dimension, we scan the time series with a fixed time window size and calculate the fractal dimension within each window. To calculate the fractal dimension, the slope of the best fit line to the linearly binned logarithmic plot of the power spectrum density versus the frequency is calculated. To achieve a stable fit, we have binned the logarithmic frequency axis by averaging the power within each bin. The slope of the best fit line $(-\beta)$ is related to the fractal dimension and the Hausdorff measure by (Turcotte 1997)

$$
\beta=2 H a+1=5-2 D .
$$

The measured $D$ is assigned to the centre of the moving time window.

\subsection{Variance fractal dimension method (VFD)}

This method assumes that the variance of incremental amplitudes of the seismic traces is scale-independent. The method basically computes the variance for differently resampled time series of the original time series and relates it to the Hausdorff measure, $\mathrm{Ha}$. Assuming a time series $B$ (with $N$ samples and sampling distance of $\delta t$ ), the increase of the signal amplitude in the sample $s\left(b_{j s}\right)$ is calculated as

$$
b_{j s}=B_{j+s}-B_{j},
$$

where $B_{j}$ and $B_{j+s}$ are amplitudes of the signal at $j$ and $j+s$ samples, respectively. Sample variance $\left(\sigma_{f s}^{2}\right)$ is calculated as

$$
\sigma_{f s}^{2}(s)=\frac{1}{n-1} \sum_{j=1}^{n}\left(b_{j s}-\bar{b}_{s}\right)^{2},
$$

where $\bar{b}_{s}$ is an average of the resample series by a sampling distance of $s \cdot \delta t$ and $n$ is the number of samples in the resample series. The upper and the lower limits for $s$ are the sampling interval and one fourth of the size of the moving window, respectively. The slope of the fitted line, $\alpha$, in the logarithmic plot of the sample variance against the $s$, is related to twice the Hausdorff measure (Jiao and Moon 2000), Ha, and is related to the fractal dimension, $D$, by

$$
D=2-\frac{\alpha}{2}=2-H a .
$$

The measured $D$ is assigned to the centre of the moving time window.
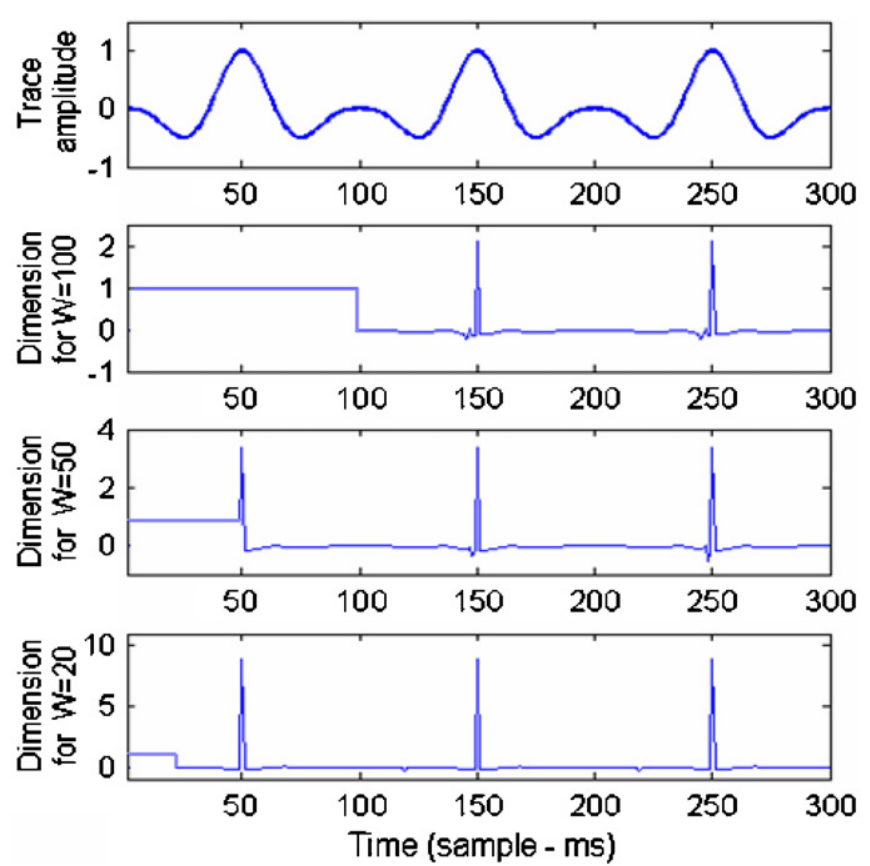

Figure 1. Finding the suitable window size for the divider method when using the instantaneous phase of a seismic trace with three zero phase wavelets. The first panel shows the seismic trace. Other panels show the fractal dimensions for different moving window sizes $W$. The sample interval is $1 \mathrm{~ms}$.

\section{Estimating the most efficient window size and the corresponding fractal dimension method and attribute}

In order to find an optimum window size for each combination of fractal dimension method and the corresponding seismic instantaneous attribute, we initialized a synthetic seismic trace containing three reflectors. This synthetic trace is the product of convolution of reflectivity series with a Ricker zero phase wavelet of dominant frequency of $10 \mathrm{~Hz}$. The sample interval was one millisecond ( $1 \mathrm{~ms})$. Three instantaneous attributes were calculated, namely instantaneous amplitude, phase, and frequency. All fractal dimension methods were used on the seismic trace and on each of the attributes mentioned in the previous section. Comparing the results, the suitable window size and corresponding optimal fractal dimension method were determined. Next, we applied this procedure to a synthetic seismic trace with a minimum phase wavelet with dominant frequency of $10 \mathrm{~Hz}$.

Since the effect of the window size is different in each fractal dimension method, the suitable window size for each method is evaluated separately. Figures 1 and 2 show the divider and Hurst fractal dimensions of, respectively, the instantaneous phase attribute of the synthetic trace with zero phase wavelet, using windows of 100, 50, and 20 samples. As mentioned in the previous section, each moving window has only one fractal dimension value. In the divider and Hurst methods this value is assigned to the last sample of the moving window, thus longer window sizes cause more data to be excluded in the beginning of the trace. Because 

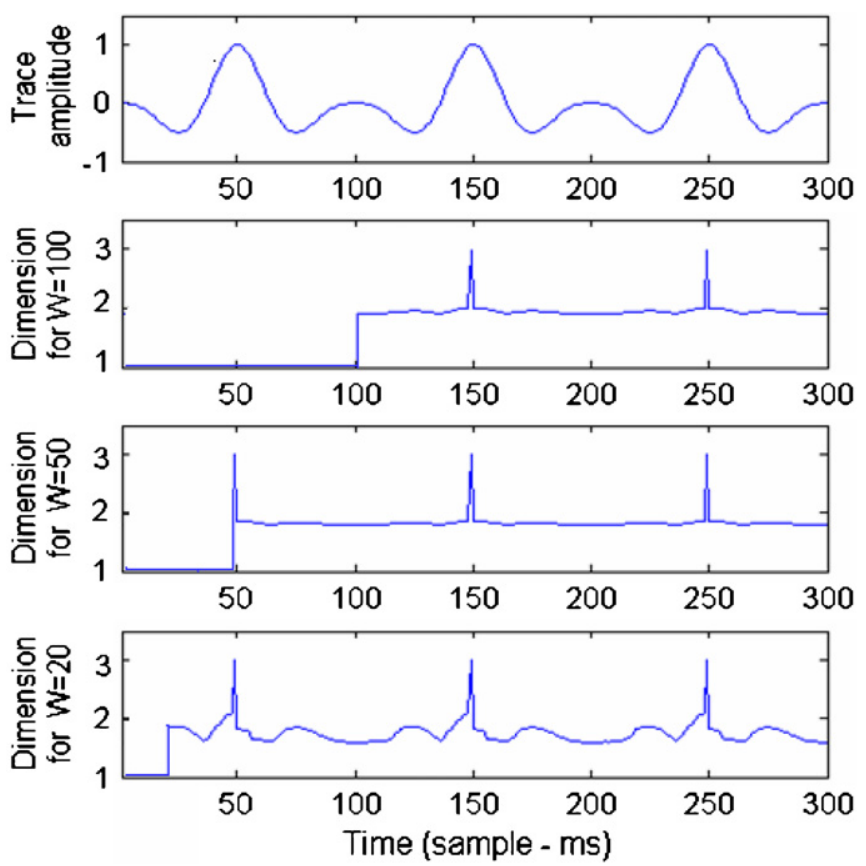

Figure 2. Same as figure 1, but for the Hurst method.
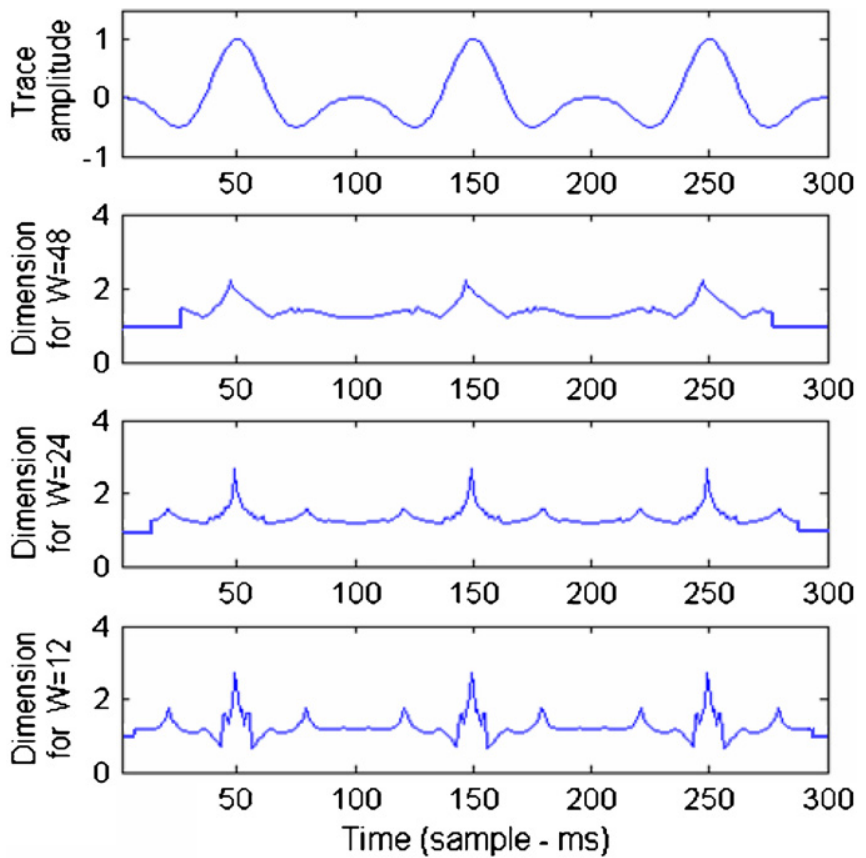

Figure 3. Same as figure 1, but for the spectral method.

the sliding window moves from left to right, the data loss occurs on the left part of the trace. For $W=100$, the first reflector falls within the missing data part. For computational reasons, this missing part is set to have a value of one. Figures 3 and 4 show the spectral and variance fractal dimensions respectively, of instantaneous phase of the synthetic trace, using window sizes of 48, 24 and 12 samples. Since the value of the fractal dimension, calculated by the spectral and variance fractal dimension methods, is assigned
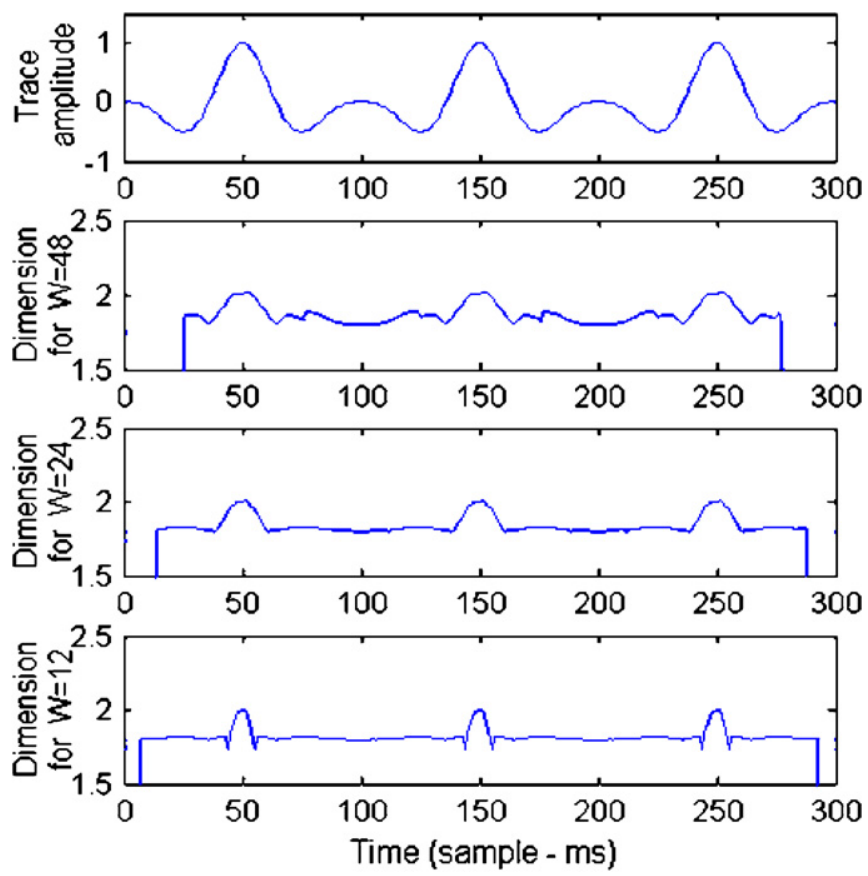

Figure 4. Same as figure 1, but for the VFD method.

to the middle of the moving window, when a window length of $\mathrm{W}$ is used, the first $W / 2$ and the last $W / 2$ samples of the trace are set to one.

In the divider method, decreasing the window size causes exaggeration to the fractal dimension. In the Hurst method, decreasing the window size causes the fractal dimension to be wavier. In the spectral and variance fractal dimension methods, smaller window sizes produce better results, but not as good as those of the divider and Hurst methods. We have found that suitable window sizes for the divider, Hurst, spectral and VFD methods are 50, 50, 24 and 12 samples, respectively. Figures 1-4 indicate that all four methods for all window sizes tested (except for $W=100$ ) can detect the reflection interfaces.

Figure 5 shows fractal dimensions calculated by the four different methods and their corresponding optimum window sizes. The divider and Hurst methods describe the reflector positions more precisely and clearly than the spectral and VFD methods. The divider method is more difficult to implement and computationally more demanding than the Hurst method. Hence, the Hurst method, using a 50 sample window, is selected as the most efficient method. Figure 6 shows the fractal dimensions of the amplitude, the instantaneous amplitude, phase and frequency of the trace, calculated by the Hurst method and a 50 sample window length. Unlike the fractal dimension of the seismic trace, the fractal dimension of the instantaneous phase detects accurately the positions of the reflectors.

A similar procedure was followed using a synthetic trace with a minimum phase wavelet of dominant frequency of $10 \mathrm{~Hz}$. We found the optimal window size for each method, the corresponding efficient suitable fractal dimension method and the instantaneous attribute that gives better results. No 

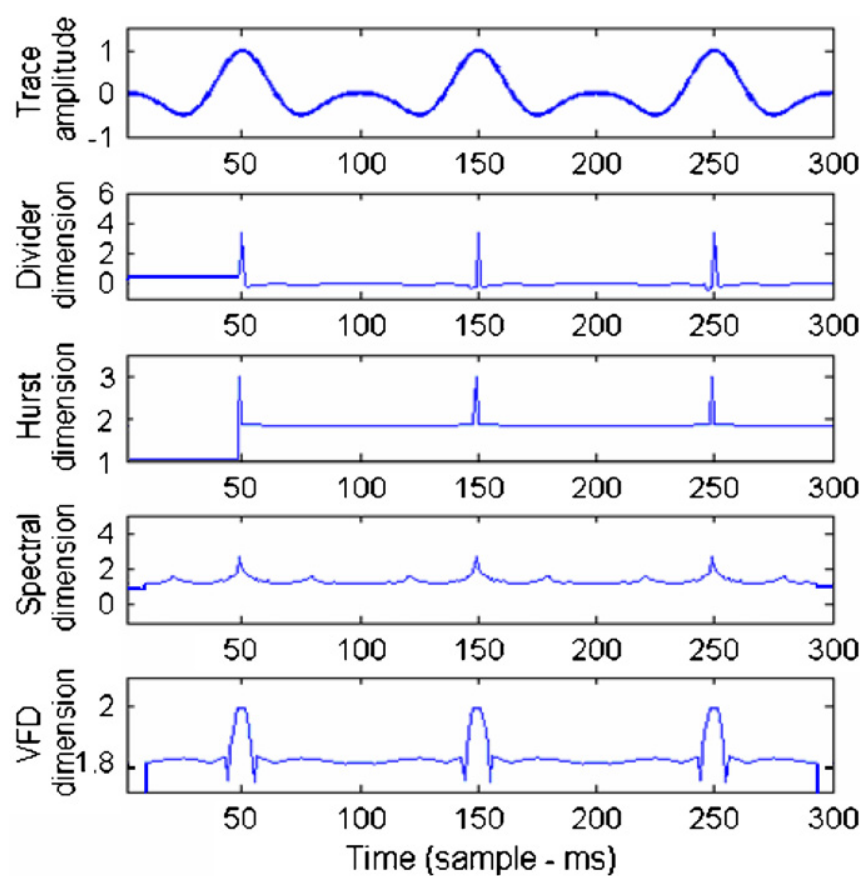

Figure 5. Finding the suitable fractal method when using the instantaneous phase with an optimum window length for each method. The first panel shows the seismic trace with three zero phase wavelets. Other panels show the fractal dimensions calculated by different fractal methods.
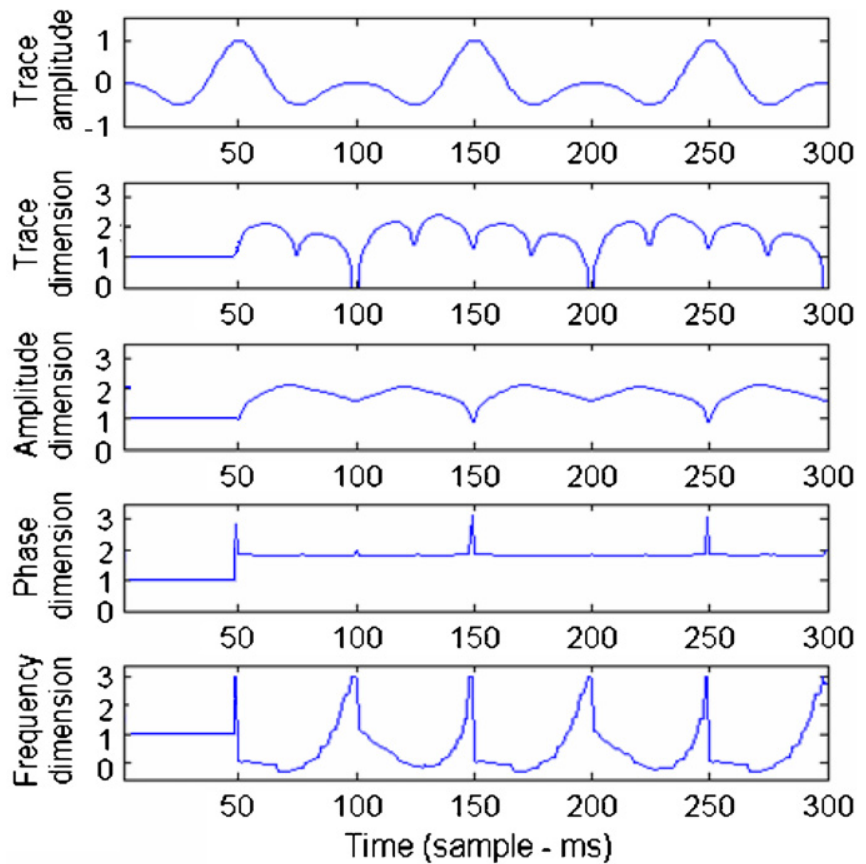

Figure 6. Finding the suitable instantaneous attribute when using the Hurst method with a window length of 50 samples. The first panel shows the seismic trace with three zero phase wavelets. Other panels show the fractal dimensions for the trace itself and its instantaneous attributes.

major differences were found by using the zero phase wavelet, in comparison with tests on the minimum phase wavelet.
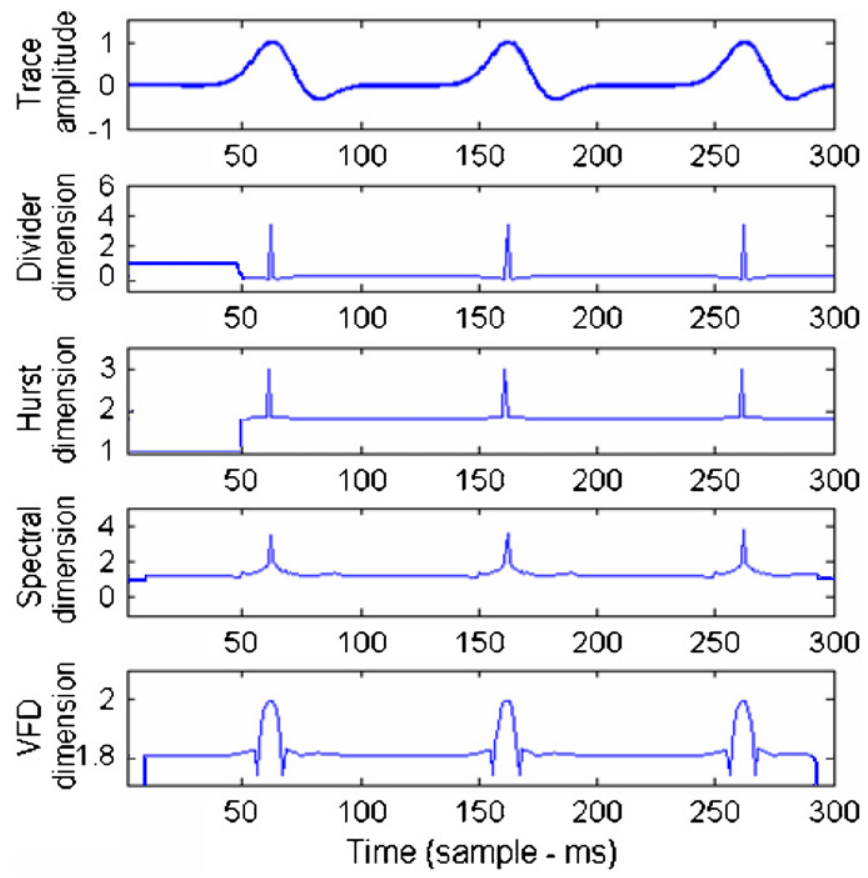

Figure 7. Same as figure 5, but for minimum phase wavelets.

We conclude that the Hurst method applied on a 50 sample window length was found to be the most appropriate method for both types of wavelets, and instantaneous phase the most suitable attribute. Therefore, we present only the comparison of the four fractal methods with their appropriate window sizes applied on the instantaneous phase attribute of a minimum phase wavelet (figure 7).

It seems that the 50 sample window length is the most efficient window size for all types of wavelets. This is because two major types of wavelets used in the seismic exploration were tested here and usually in the seismic processing other types of wavelets are converted to zero or minimum phase wavelets. When the dominant frequency of the wavelet decreases, its dominant wavelength increases and a longer duration window must be used to calculate the fractal dimension (Nath and Dewangan 2002). The range of frequencies encountered in seismic exploration is usually 10$100 \mathrm{~Hz}$, thus we expect the 50 sample window length, with $1 \mathrm{~ms}$ sampling interval, to yield good results in this range. For different sampling intervals the window size must be adapted accordingly.

As described above, in the Hurst method we do not calculate an average $R$ and $S$ for all time window segments, instead we calculate the $R$ and $S$ values for the last time window segment within each moving time window. This makes the Hausdorff measure in our algorithm very sensitive to the scaling of the signal at the end of a moving window and less sensitive to the other parts, especially at the beginning of it. Hence the window size in our algorithm does not affect our results much, as is obvious in figure 2, where both window sizes of 20 and 100 samples show the wavelets as well. 

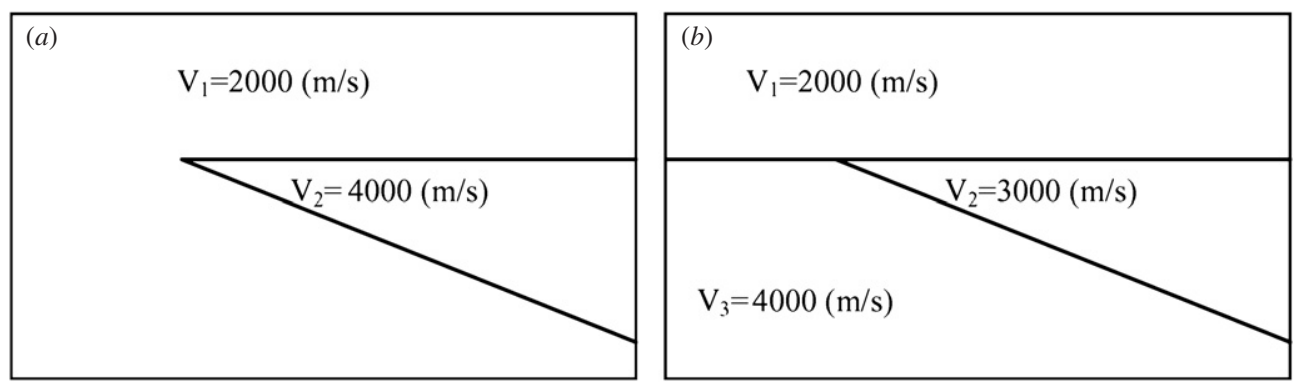

Figure 8. Wedge models used to generate the synthetic seismic sections in figures 9-12. Assuming constant density for all layers: (a) the reflection coefficient of the upper wedge interface is positive and the lower interface negative, whereas in $(b)$ the polarities are positive.


Figure 9. Convolution of the wedge model in figure 8(a) with the zero phase Ricker wavelet with a dominant frequency of $10 \mathrm{~Hz}$. The left panels from top to bottom show the 2D synthetic seismic sections with a S/N ratio of infinity (noiseless), 10, 6 and 2, respectively. The right panels show the corresponding fractal dimensions of the instantaneous phase for the left panels. The fractal dimensions are calculated using the Hurst method with a window length of 50 samples and a sampling interval of $1 \mathrm{~ms}$. The interference is generally detectable at and beyond the 7 th trace. On the 7 th trace, the distance between the top and bottom reflectors is equal to $\lambda / 16$. Decreasing the $\mathrm{S} / \mathrm{N}$ ratio generates multiple peaks for each reflector, which in turn limits our ability to resolve the interfered seismic wavelets. 

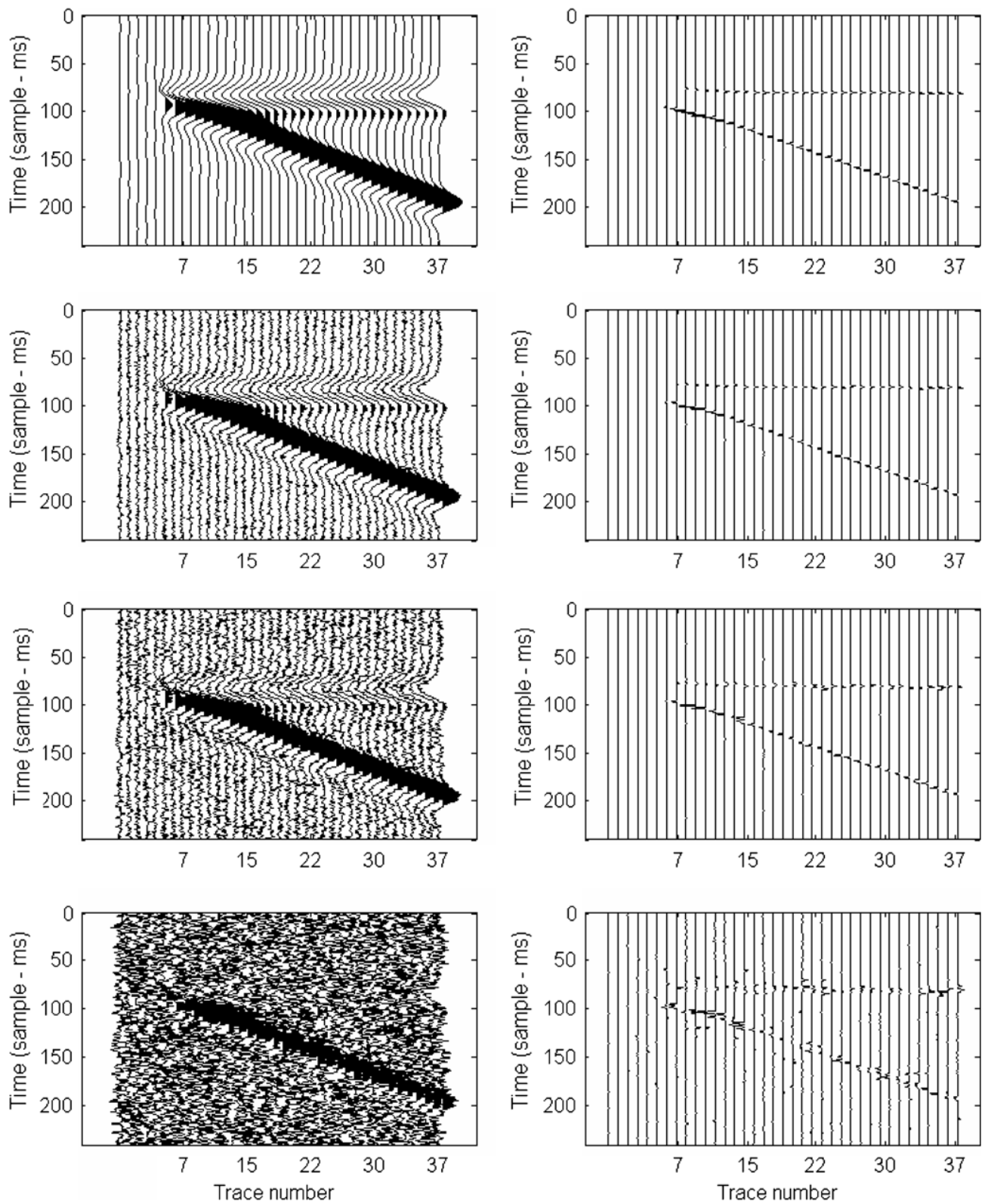

Figure 10. Same as figure 9, but for a minimum phase Ricker wavelet with a dominant frequency of $10 \mathrm{~Hz}$.

\section{Two-dimensional synthetic seismic sections}

The most suitable fractal method (the Hurst method) and attribute (instantaneous phase) are now used to confirm the effectiveness of the fractal methods for separating interfering wavelets. In order to study wavelets interfering in 2D seismic sections, we generate four synthetic seismic sections, by convolving zero- and minimum-phase Ricker wavelets with two wedge models (figure 8). Assuming constant density for all layers, only the velocities affect seismic impedances. In the first model, figure $8(a)$, the reflection coefficient of the upper wedge interface is positive and the lower interface negative whereas for the second model, figure $8(b)$, the polarities are positive. In this study, we use the 1D instantaneous phase attribute of each trace of the 2D synthetic sections. The fractal dimension of the instantaneous phase of each trace of the seismic sections is calculated using the Hurst method with a 50 -sample moving time window with $1 \mathrm{~ms}$ sampling intervals.

Figures 9-12 show the synthetic seismic sections of the wedge models of figure 8 with four different signal-to-noise $(\mathrm{S} / \mathrm{N})$ ratios (the left panels) and their associated fractal dimension results (right panels). In these figures, the top first row is noiseless, whereas the remaining rows from top to bottom contain $\mathrm{S} / \mathrm{N}$ ratios of 10,6 , and 2, respectively. Figures 9 and 10 are the result of convolution of the wedge model of figure $8(a)$ with the zero- and minimum-phase wavelets. Figures 11 and 12 are the same as figures 9 and 10 but based on the model in figure $8(b)$. From figures $9-12$ it is clear that $\mathrm{S} / \mathrm{N}$ ratios equal or greater than 10 have insignificant effect on the results of the fractal dimension distributions. Decreasing the $\mathrm{S} / \mathrm{N}$ ratio generates more than one peak for 

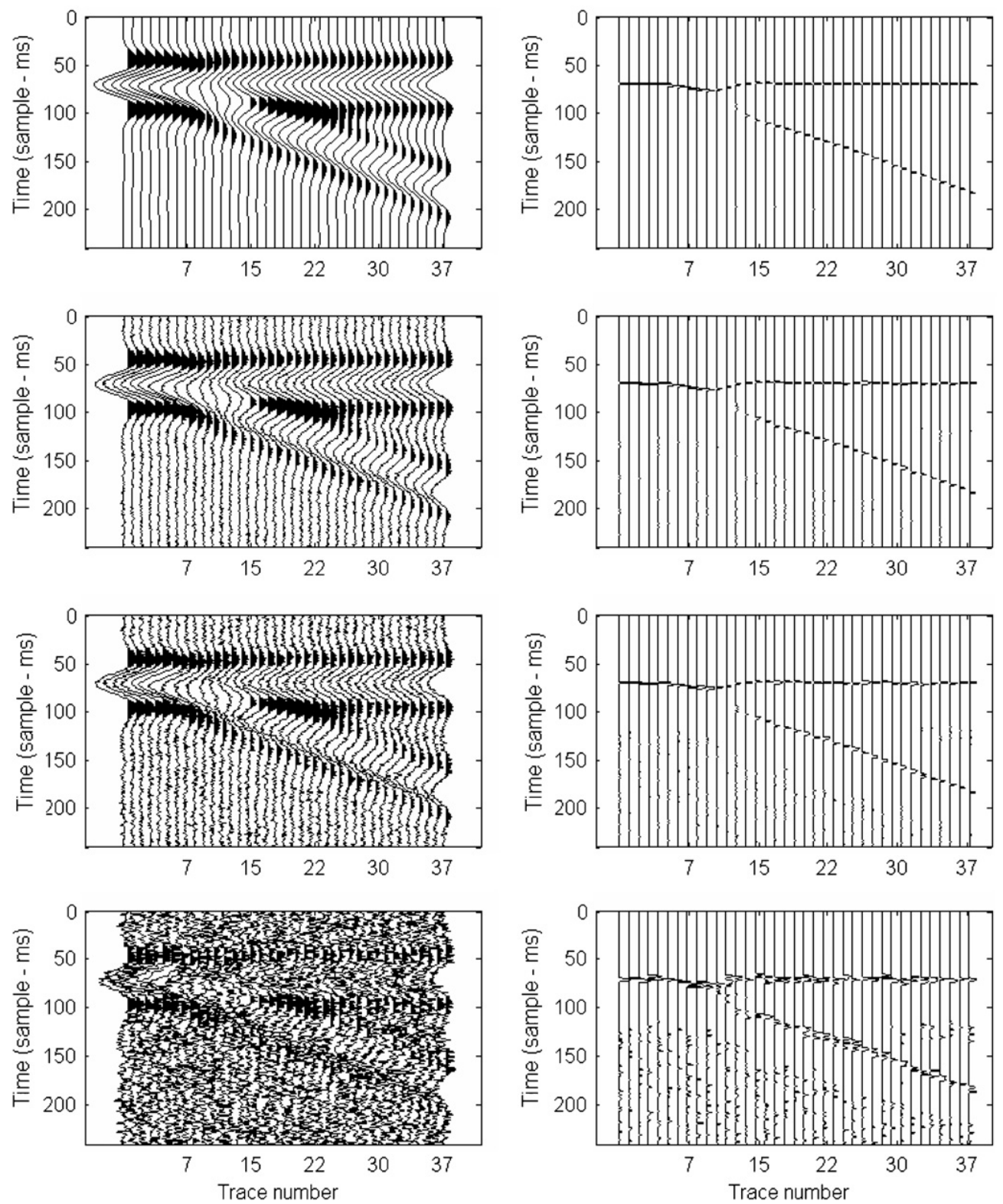

Figure 11. Convolution of the wedge model in figure $8(b)$ with the zero-phase Ricker wavelet with a dominant frequency of $10 \mathrm{~Hz}$. The left panels from top to bottom show the 2D synthetic seismic sections with a $\mathrm{S} / \mathrm{N}$ ratio of infinity (noiseless), 10, 6 and 2, respectively. The right panels show the corresponding fractal dimensions of the instantaneous phase for the left panels. The fractal dimensions are calculated using the Hurst method with a window length of 50 samples and a sampling interval of $1 \mathrm{~ms}$. The interference is generally detectable at and beyond the 13th trace. On the 13th trace, the distance between the top and bottom reflectors is equal to $\lambda / 4$. Decreasing the $\mathrm{S} / \mathrm{N}$ ratio generates multiple peaks for each reflector, which in turn limits our ability to resolve the interfered seismic wavelets.

each reflector; hence, the position of each reflector cannot be determined correctly. As shown in the figures $9-12$, the $\mathrm{S} / \mathrm{N}$ ratio of 6 has little effect on the results and does not diminish the coherency of the peaks along the reflectors. The presence of random noise and its effects on detecting seismic reflectors are similar for both minimum- and zero-phase wavelets as shown in figures 9-12. In all noisy sections, scattered peaks are detected in the fractal dimension distribution, where no wavelet is presented. These peaks are related to low $\mathrm{S} / \mathrm{N}$ ratios in regions where the signal amplitude is very small.

Each section contains 37 traces. The distance between the reflected wavelets from the upper and the lower interfaces increases linearly from zero at the 5 th trace to one wavelength at the 37 th trace. Therefore, we have distances of $\lambda / 16, \lambda / 8$, $\lambda / 4, \lambda / 2$, and $\lambda$ at the 7 th, 9th, 13th, 21st and 37th traces, respectively. In figures 9 and 10, the interference is detectable from the 7 th trace. Hence, by this method, the threshold for detecting wavelet interference is $\lambda / 16$ when the reflection coefficient of the upper wedge interface is positive and the lower interface negative. Similarly, in figures 11 and 12, the interference is detectable from the 13th trace. Hence, by this method, the threshold for detecting wavelet interference is $\lambda / 4$ when the reflection coefficients of the upper and the lower interfaces of the wedge are positive. 

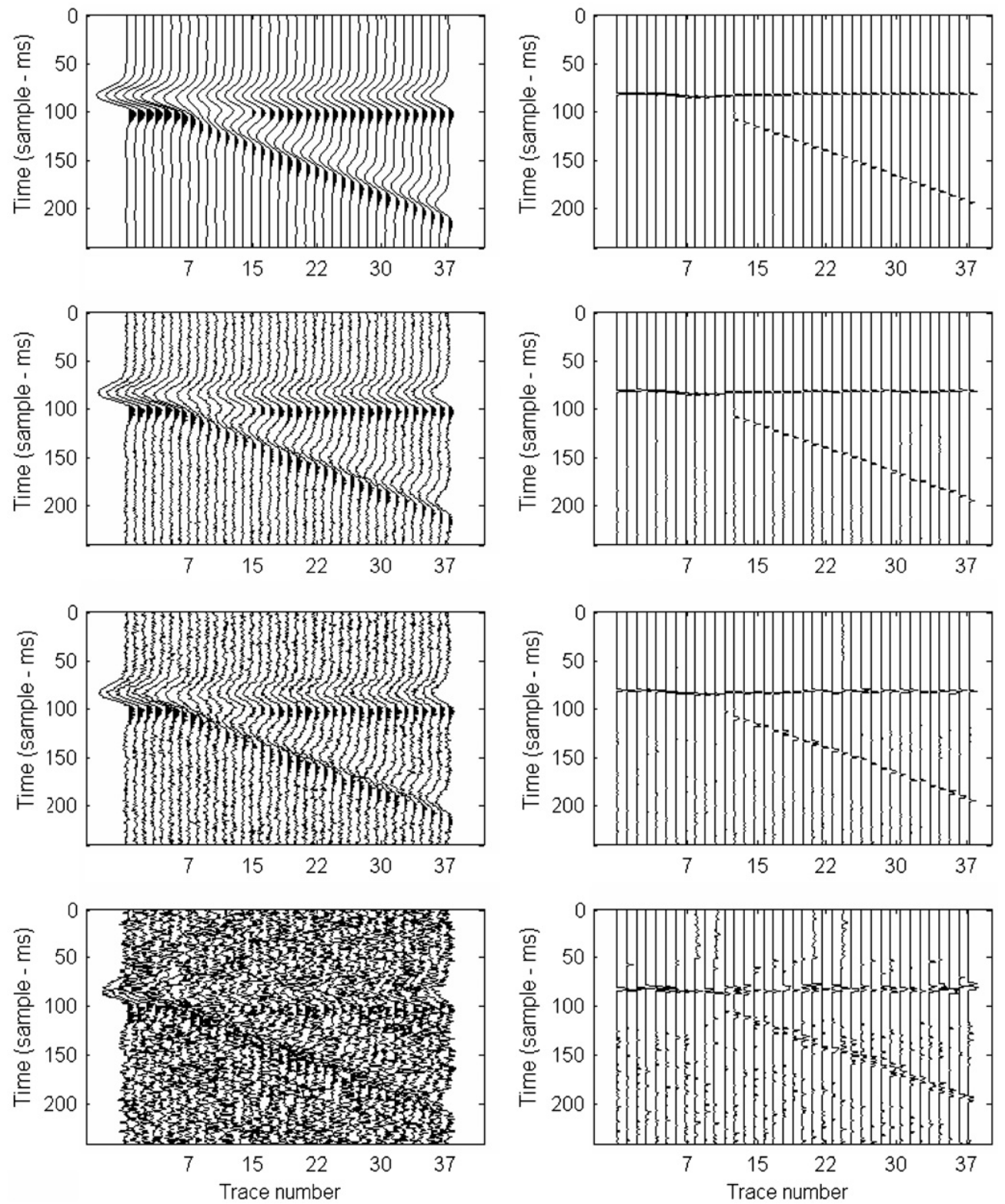

Figure 12. Same as figure 11, but for a minimum phase Ricker wavelet with the dominant frequency of $10 \mathrm{~Hz}$.

\section{Conclusions}

We have applied four fractal methods (divider, Hurst, spectral, and variance fractal dimension) to a seismic trace and its three instantaneous attributes (amplitude, phase and frequency) to find the optimum fractal method and the appropriate seismic attribute. We have found that the Hurst fractal dimension method, with a 50-sample moving window, is the best fractal method-attribute combination. We have evaluated the sensitivity of the proposed fractal method in detecting seismic reflectors on a series of synthetic seismic wedge models, using two types of wavelets (zero phase and minimum phase) and different random noise levels.

We have found that the fractal analysis of the instantaneous phase, using the Hurst method, can separate wavelets having a distance of $\lambda / 16$ or greater when the reflection coefficient of the upper wedge interface is positive and the lower interface negative; when the polarities are positive, the proportional distances become $\lambda / 4$ or greater. Our results are not sensitive to random noise provided that the signal-to-noise ratio is greater than 10 . Decreasing the signalto-noise ratio generates multiple peaks for each reflector, which in turn limits the ability to separate in separating interfered seismic wavelets.

\section{Acknowledgments}

We greatly appreciate the constructive and thoughtful comments of the anonymous reviewers. We thank Nikos Economov from the Laboratory of Applied Geophysics, Department of Mineral Resources Engineering, Technical University of Crete, Greece, Mohammad Forman Asgharzadeh and Bahman Farnudi for their suggestions for 
improving the writing style. The research on which this paper is based was supported by the Institute of Geophysics, University of Tehran under grant 6201004/1/01.

\section{References}

Barnes A E 1993 Instantaneous spectral bandwidth and dominant frequency with applications to seismic reflection data Geophysics 58 419-28

Boschetti F, Dentith D and List R D 1996 A fractal-based algorithm for detecting first arrivals on seismic traces Geophysics 61 1095-102

Gholamy Sh, Javaherian A and Ghods A 2006 Automatic detection of wavelets interfering in seismic sections using fractal methods Proc. 8th SEGJ Int. Symp.Imaging and Interpretation (Kyoto, Japan, 26-28 Nov.)

Hayward J, Orford J D and Whalley W B 1989 Three implementations of fractal analysis of particles outlines Comput. Geosci. 15 199-207

Hurst H E, Black R P and Simaika Y M 1965 Long-Term Storage (London: Constable Press)
Jiao L and Moon W M 2000 Detection of seismic refraction signals using a variance fractal dimension techniques Geophysics 65 286-92

Kaye B H 1989 A Random Walk Through Fractal Dimension (New York: VCH)

Kennedy S K and Lin W 1986 FRACT-A fortran subroutine to calculate the variables necessary to determine the fractal dimension of closed forms Comput. Geosci. 12 705-12

Mandelbrot B B 1967 How long is the coast of Britain? Statistical self-similarity and fractional dimension Science 156 636-8

Mandelbrot B B 1985 Self-affine fractals and fractal dimension Phys. Scr. 32 257-60

Meissner R and Meixner E 1969 Deformation of seismic wavelets by thin layers and layered boundaries Geophys. Prospect. 17 1-27

Nath A K and Dewangan P 2002 Detection of seismic reflection from seismic attributes through fractal analysis Geophys. Prospect. 50 341-60

Russ J C 1994 Fractal Surfaces (New York: Plenum)

Taner M T, Koehler F and Sheriff R E 1979 Complex seismic trace analysis Geophysics 44 1041-63

Turcotte D L 1997 Fractals \& Chaos in Geology \& Geophysics 2nd edn (Cambridge: Cambridge University Press) 